\title{
Prognostic Factors in Obturator Hernia
}

\author{
Semra Salimoğlu ${ }^{1}$, Semra Demirli Atıcl, ${ }^{1, *}$
}

\author{
${ }^{1}$ University of Health Sciences Tepecik \\ Training and Research Hospital, \\ Department of General Surgery, İzmir, \\ Turkey
}

\section{*Correspondence}

smrdemirli@hotmail.com

(Semra Demirli Atıcı)

\begin{abstract}
Objective: Obturator hernia is a rare type of hernia that can occur in the octogenarian patients. We discuss the diagnosis, treatment and management of patients with obturator hernia, and describe the clinical presentation, laboratory and radiological findings. Material and methods: A retrospective study was performed in ten patients diagnosed with an obturator hernia between December 2008 - 2019 at a single tertiary hospital. Patients' demographic features, clinical symptoms, laboratory and imaging data, comorbidities, surgical treatment methods, postoperative morbidity and mortalities were evaluated. Results: The patients' mean age was 84.4 years (range between 81 - 89 years). All patients presented with symptoms of small bowel obstructions; abdominal pain located in the lower quadrant $(100 \%)$, vomiting $(60 \%)$, nausea ( $80 \%)$ and distension $(60 \%)$. All patients had air-fluid levels on X-ray. We performed small intestine resection and an end to end anastomosis in four patients, resection with ileostomy in two patients and bowel reduction in four patients. Postoperatively, an anastomosis leak occurred in two patients, they were re-operated and an ileostomy was performed. In the post-op period, six patients died, and four patients survived. Conclusion: Obturator hernia is seen in octogenarian patients with symptoms of small bowel obstruction. The diagnosis is based mostly on computed tomography findings. An elevated neutrophil-lymphocyte ratio, platelet-lymphocyte ratio and lactate levels can be used as a biomarker for the prediction of non-viable small bowel in these $\mathrm{OH}$ patients.
\end{abstract}

\section{Keywords}

Obturator hernia, Octogenarian, Neutrophil-to-lymphocyte ratio, Platelet-to-lymphocyte ratio

\section{Introduction}

Obturator hernia $(\mathrm{OH})$ is a rare type of hernia, that occurs as a result of the protrusion of intraperitoneal or extraperitoneal organs or tissues through the obturator canal [1]. OH was first described in 1724 by Arnaud de Ronsil [1, 2]. The incidence of $\mathrm{OH}$ is between 0.05 and $1.4 \%$ of all hernias and occurs predominantly in elderly, malnourished, multiparous, female patients due to a wider pelvis, greater transverse diameter [13].

The most significant clinical manifestation of $\mathrm{OH}$ is abdominal pain, vomiting and nause due to small bowel obstruction $[3,4]$. The mortality rate of $\mathrm{OH}$ can be as high as $70 \%$ due to delayed diagnosis and the need for surgical intervention in these elderly patients with comorbid diseases.

In this retrospective study, we aimed to evaluate the diagnosis, treatment and management of patients with an obturator hernia, and describing the clinical presentation, laboratory and radiological findings and the outcomes following surgery in these high risk patients.

\section{Materials and methods}

Patients admitted to the emergency department of a tertiary hospital with acute abdominal pain who eventually underwent a surgical operation due to an obturator hernia between December 2008 and December 2019 were included in the study. Demographics, as well as Body Mass index (BMI), age, sex, duration of symptoms, Howship-Romberg sign, co-morbid diseases, findings from physical examination, intraoperative findings, surgical treatment types, repairs with and without prostethic mesh, preoperative imaging studies and laboratory results, preoperative arterial blood samples for lactate levels, preoperative neutrophil/lymphocyte and lymphocyte/platelet levels based on laboratory results, duration of hospitalization, postoperative morbidity and mortality were recorded. Due to the small size of the current patient group, no comparison statistical testing was performed. Laboratory value were evaluated by studying statistical mean values. For this descriptive observational study voluntary informed consent was obtained from each of the patients, and they were informed that their information would be used for publication. Approval from University of Health Sciences Tepecik Training and Research Hospital ethics board was obtained. 


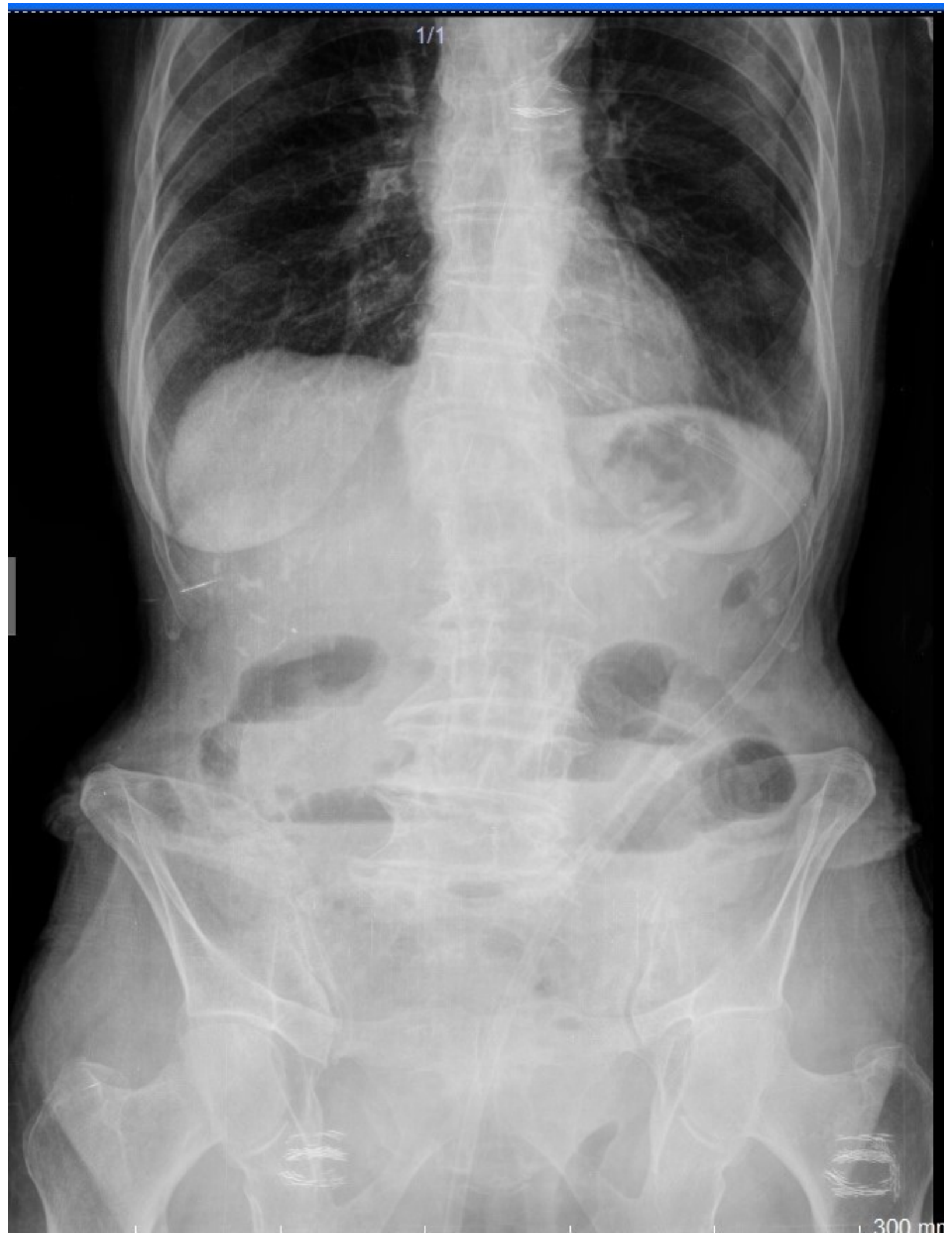

F I G U R E 1. Abdomen X-Ray shows air fluid levels.

\section{Results}

All ten patients were female and octogenarian without a history of abdominal surgery. The patients' mean age was 84.4 years (range between 81 - 89 years). The mean time from the beginning of symptoms to the presentation was 4.4 days (ranged from 2 - 6 days). Ten patients presented with symptoms of intestinal obstructions; abdominal pain located in the lower quadrant $(100 \%)$, vomiting (60\%), nausea $(80 \%)$ and distension $(60 \%)$. A Howship-Romberg sign was positive in four patients $(40 \%)$ who were admitted to the emergency service at least two times [1-3] with hip pain.

Co-morbid diseases included chronic obstructive lung disorder $(80 \%)$, chronic heart failure $(60 \%)$, hypertension $(80 \%)$, 


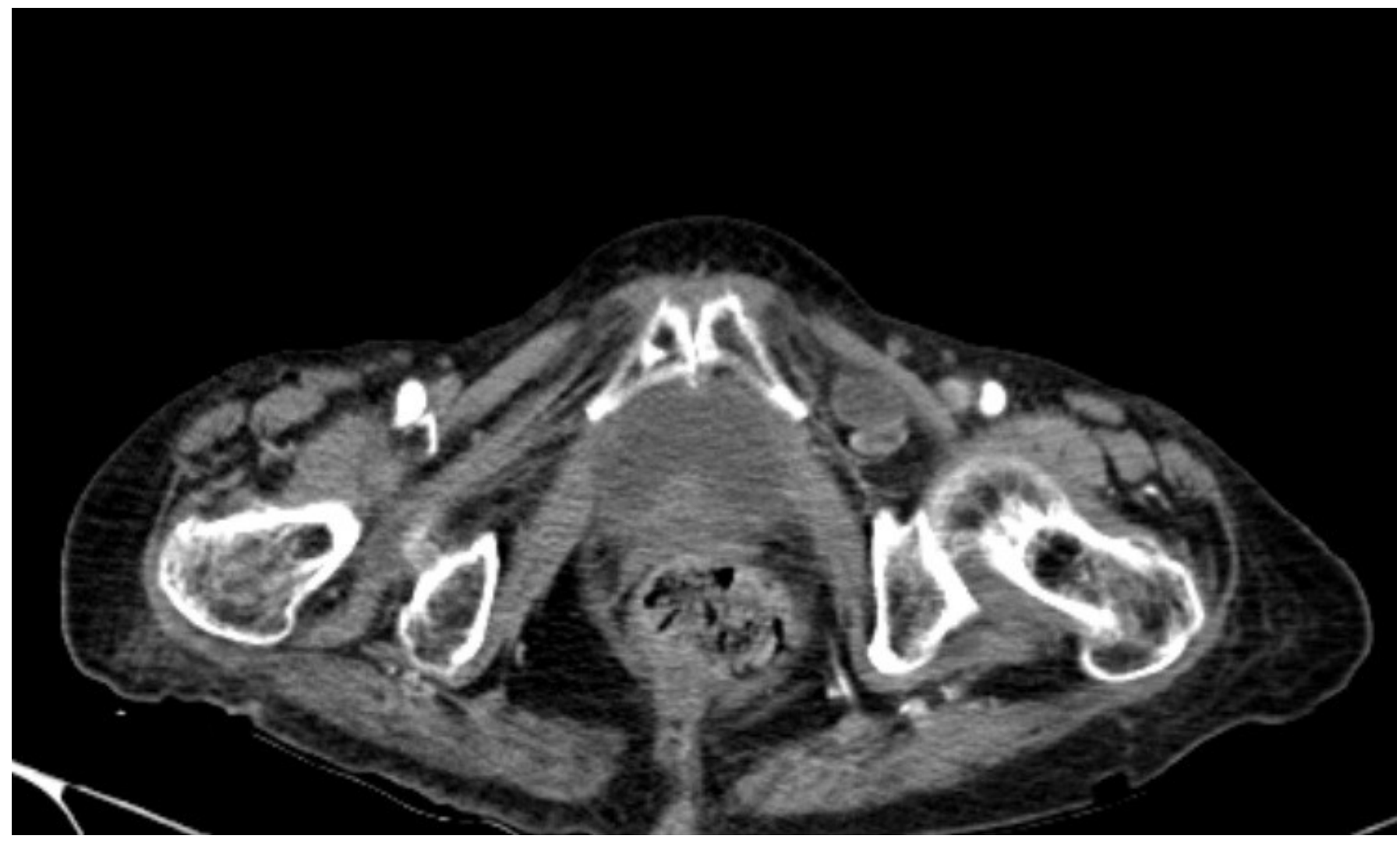

F I G U R E 2. CT shows intestinal loop in the left obturator canal.

coronary artery disease (40\%), rheumatoid arthritis (20\%), cerebrovascular disease (20\%), and cardiac arrhythmia (20\%). An abdominal CT was performed in all ten patients who had small bowel loop with air-fluid levels on a plain abdominal X-Ray (Fig. 1). The presumptive diagnosis was made preoperatively in ten patients based on CT findings (Fig. 2) and supported by perioperative findings. The mean lactate level was $5.6 \mathrm{mmol} / \mathrm{L}$ (ranged between 1.4 - 9.4mmol/L). The mean lactate level of six patients which had a non-viable small bowel was higher with a range of $7.73 \mathrm{mmol} / \mathrm{L}(5.4$ $9.4 \mathrm{mmol} / \mathrm{L})$ than four patients with viable small bowel with the range of $2.4 \mathrm{mmol} / \mathrm{L}(1.4-3.4 \mathrm{mmol} / \mathrm{L})$. The mean range of neutrophil/lymphocyte ratio was 14.86 (between 8.33 22.86 ), the mean platelet/lymphocyte ratio was 790.254 (between 219.9 - 1043.3) in four patients who had incarcerated viable small bowel due to $\mathrm{OH}$. The mean range of neutrophil/lymphocyte ratio was 24.71 (between 9.45 - 53.8), the mean platelet/lymphocyte ratio was 990.658 (between 183.3 2700 ) in six patients who had a non-viable small bowel due to $\mathrm{OH}$.

All patients underwent emergency surgery. Laparotomy was performed with a midline incision. Six patients had a left side hernia with jejunum involvement and four had a right side hernia with ileum involvement. At laparotomy, six patients had a non-viable small bowel. Small bowel resection and an end to end anastomosis was performed in four patients, resection with an ileostomy was performed in two patients. In four patients, the hernia defects were repaired with prostethic mesh, and in six patients, the hernia defect was repaired with nonabsorbable suture. In two patients with a strangulated hernia, a small bowel resection with an end ileostomy was performed due to fecal peritonitis and the defect was repaired by non-absorbable suture without prostethic mesh. In four patients with viable small bowel, the bowel was reduced and the defect was repaired with non-absorbable suture and prostethic mesh. Postoperatively, patients remained in the intensive care unit for 1 - 39 days. A postoperative anastomosis leak occurred in two patients, they were re-operated and an ileostomy was performed. Six patients died and four survived. One patient who had fecal peritonitis due to perforation of non-viable small bowel died on the second postoperative day due to septic shock. Six of five patients who had chronic obstructive lung disorder died due to the pneumonia on the 29th, 39th, 37th and 34 th postoperative day.

\section{Discussion}

Obturator hernia $(\mathrm{OH})$, is an uncommon clinical entity in octagenarians and is difficult to diagnose [1]. $\mathrm{OH}$ is an abdominal hernia generally seen in elderly, scraggy, multiparous woman $[1,2]$. $\mathrm{OH}$ has been associated with multiple co-morbidities including advanced age, malnutrition, raised intra-abdominal pressure due to ascites, chronic constipation, chronic obstructive pulmonary disease and kyphoscoliosis [2-4]. $\mathrm{OH}$ is nine times more common in women than men due to a broader pelvis, a larger obturator canal and history of pregnancy $[1,2]$. Multiple pregnancies, resulting in increased intra-abdominal pressure, cause weakening of the pelvic peritoneum and obturator membranes, which are prone to hernia development [4]. Malnutrition is a predisposing factor due to the decreased preperitoneal fat and lymphatic tissue over the obturator canal which increases the risk of herniation $[5,6]$. In addition to 


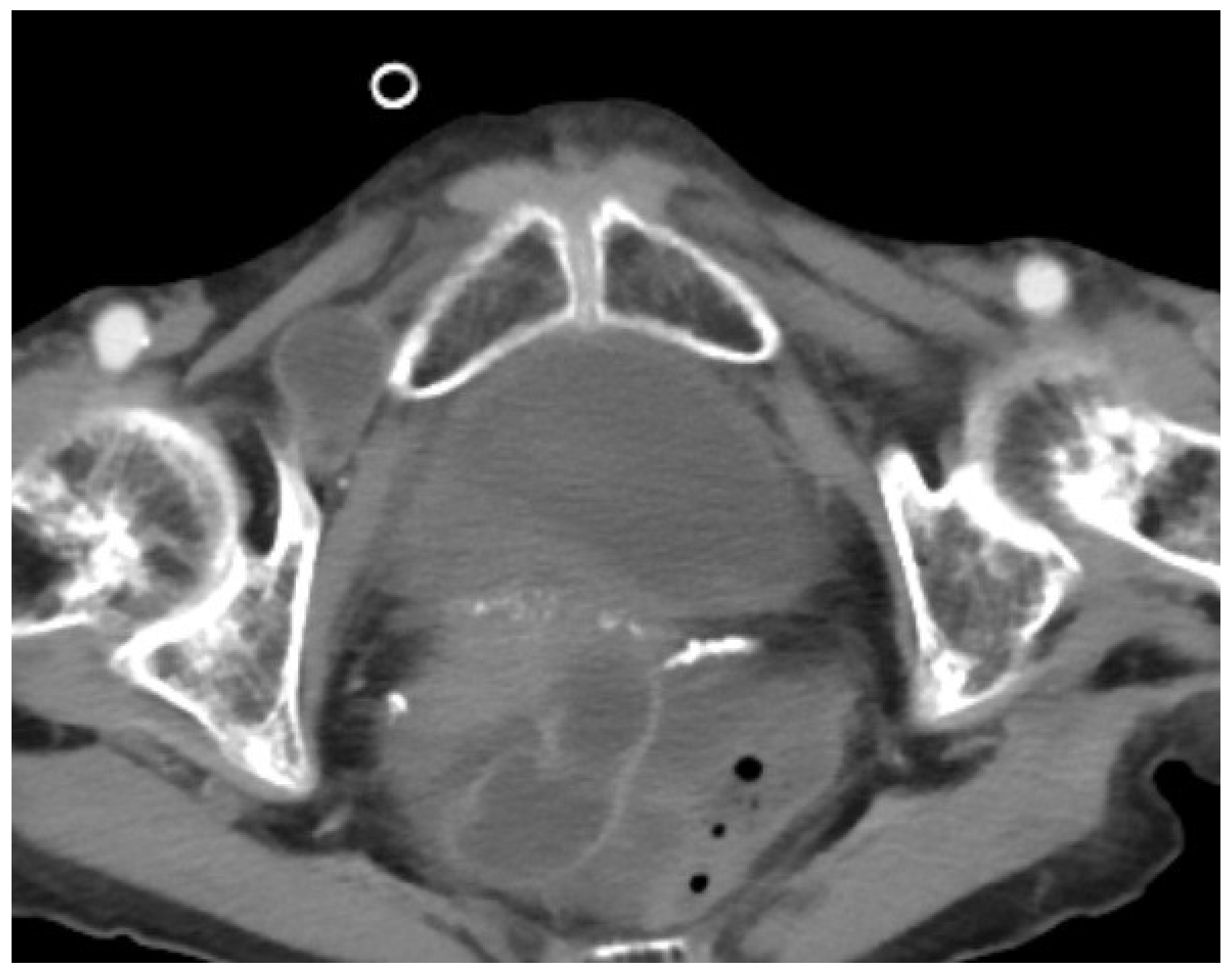

F I G U R E 3. CT shows intestinal loop in the right obturator canal.

these risk factors, Mantoo et al [7] reported, Asians have been reported to have higher rates of $\mathrm{OH}$ while Western studies indicate a much lower incidence. $\mathrm{OH}$ is generally seen in frail,elderly female patients between 70 and 90 years and has been called "little old lady's hernia"[7]. All the patients in our study were emaciated, female, octogenarian and multiparous consistent with the literature. Obturator herniation usually occurs on the right side. It is thought that in case involving the left side, the sigmoid colon covers the left obturator foramen and this may prevent hernia formation [1,2]. However, Igari et al [2] reported the incidence of $\mathrm{OH}$ was equal on both sides, Thanapaisan et al [1] reported left hernia are more common than right hernia, with a rate of $3: 2$, similar to our study. The hernia sac usually contains small bowel, especially ileum; although jejunum, colon, omentum, appendix, Meckel's diverticulum, ovary, fallopian tube, urinary bladder have all been reported in hernia sac [6].

$\mathrm{OH}$ commonly presents with symptoms of intestinal obstruction including abdominal pain, nausea, vomiting, and distention $[8,9]$. The Howship-Romberg sign is pathognomonic for $\mathrm{OH}$ and occurs due to the distribution of the obturator nerve by the pressure of the hernia sac $[8,9]$. This sign occurs by the internal rotation of the hip, and the pain is aggravated by lengthening of the thigh, abduction and medial rotation $[8,9]$. Although Thanapaisan et al [1] reported that $13 \%$ of patients had a Howship-Romberg sign, Igari et al [2] reported a 50\% positive Howship-Romberg sign similar to our study. The mean time from the beginning of symptoms to presentation was 4.4 days and ranged from 2 - 6 days in our study. The patients with non-viable bowel presented after a mean of 5.66 days (5 - 6 days), which was higher than patients with viable bowel, 2.5 days ( $2-3$ days). Chang et al [10] reported that one of the major causes of bowel resection in these patients was the duration of symptoms.

Earlier diagnosis is challenging when the symptoms and signs are nonspecific. Various imaging examinations such as ultrasonography, herniography, CT scan, and laparoscopy, have been used to establish the diagnosis. Abdominal X-Ray and abdominal ultrasonography (USG) usually reveal a pattern of intestinal obstruction. Although USG is a fast, non-invasive, cost-effective, and easily- accessible diagnostic method, it still may be difficult to make the diagnosis of an obturator hernia. Kulkarni et al [5] reported that, the best imaging tool is CT with superior sensitivity and precision compared to other noninvasive diagnostic tools. CT is minimally invasive, easily accessible and can be performed over a short time. 
The most common CT scan finding in $\mathrm{OH}$ is herniation of small bowel extending through the obturator foramen which is located between the pectineus and obturator externus muscles $[1,2]$. CT also distinguishes obturator hernia from other abdominal pathologies such as masses, tumors, hematomas, and abscesses [5]. In our study, all $\mathrm{OH}$ patients were diagnosed with an abdominal CT scan. It has been reported that the use of CT before surgery in patients with an obturator hernia reduces intestinal resection rates and mortality [8], Nasir et al. [9] reported that preoperative CT is beneficial in the definitive and early diagnosis of the disease but has no effect on the postoperative survival rate and does not increase longterm survival. Due to delayed presentation, advanced age, and the presence of comorbidities, there is often a delay in the diagnosis and surgical intervention in patients with an obturator hernia [6]. Delay in the diagnosis may occur in these elderly patients due to their comorbidities and senescence with increased dementia [6].

Neutrophil-lymphocyte ratio (NLR) and plateletlymphocyte ratio (PLR) can be a good predictor of the severity of inflammatory response [10-13]. High NLR and PLR levels can be used as a marker to estimate intestinal necrosis and the need for intestinal resection in patients who will undergo surgery for $\mathrm{OH}[11,12]$. In our study, duration of symptoms were correlated with lactate, NLR and LPR levels. Lactate levels, NLR and PLR were higher in patients requiring a bowel resection due to non-viable bowel compared to the no-bowel resection group similar to the findings of Köksal et al [11].

Lactate is a product of anaerobic metabolism of glucose, and lactate concentration in arterial blood is an important marker in demonstrating tissue hypoperfusion [13, 14]. Anaerobic metabolism causes increased lactate levels, which is predictive for non-viable bowel [13, 15]. NLR and PLR, lactate levels were higher in patients with strangulated bowel than in the incarceration group. Tanaka et al [14] reported that arterial blood lactate level $(2.0 \mathrm{mmol} / \mathrm{L}$ or greater $)$ is a determinative factor for non-viable bowel. The mean lactate level of six patients which had a small bowel strangulation was 7.73 $\mathrm{mmol} / \mathrm{L}(5.4-9.4 \mathrm{mmol} / \mathrm{L})$ higher than the four patients with incarceration of small bowel with the range of $2.4 \mathrm{mmol} / \mathrm{L}$ ( 1.4 - 3.4mmol/L).

Although, a non-surgical method for the treatment of $\mathrm{OH}$ has been reported in the literature, surgery remains the most definitive treatment for $\mathrm{OH}[2,16]$. Various surgical approaches and methods have been reported in the literature such as the abdominal, retropubic, obturator, inguinal and the laparoscopic approach in the past two decades $[1,2]$. Although the laparoscopic approach is minimally invasive and is associated with less postoperative pain, less pulmonary complications and a shorter hospitalization stay for these highrisk patients, the abdominal approach is preferred in an urgent situation [8, 9].

Although surgeons have used various techniques to close the defect such as costal cartilage, the innermost fibres of pectineus muscle, an osteoperiosteal flap from the pubic bone, omentum and uterine fundus or round ligament, currently, the defect is generally closed diredtly using sutures, or with tissue flaps, or prosthetic mesh $[9,10]$. In our study, we used an open surgical approach in all cases. The defect was closed with nonabsorbable suture in six patients; and prostethic mesh was used in for patients.

\section{Conclusion}

Obturator hernia can be difficult to diagnose due to nonspecific presentation in octogenarian, thin, female patients. Although CT scan is an effective and beneficial diagnostic tool in the diagnosis of $\mathrm{OH}$, an elevated NLR, PLT and lactate levels can be used as a biomarker for the prediction of non-viable bowel and bowel resection due to $\mathrm{OH}$.

\section{Limitations}

Our study is retrospective, descriptive and had a small group of patients. Studies with a larger number of patients are needed to determine the most optimal diagnostic methods and surgical techniques to improve survival in patients with $\mathrm{OH}$.

\section{ACKNOWLEDGEMENTS}

I would like to express my gratitude to Professor Cengiz Aydın and Tayfun Kaya for advices drafting the study and on statistical analysis. We thank our colleagues from University of Health Sciences Tepecik Training and Research Hospital, Department of General Surgery who provided insight and expertise that greatly assisted the research. Thanks to all the peer reviewers and editors for their opinions and suggestions.

\section{CONFLICT OF INTEREST}

We declare that we do not have any commercial or associative interest that represents a conflict of interest in connection with the work submitted.

\section{REFERENCES}

[1] Thanapaisan C, Thanapaisal C. Sixty-One Cases of Obturator Hernia in Chiangrai Regional Hospital: Retrospective Study. J Med Assoc Thai. 2006;89:2081-2085.

[2] Igari K, Ochiai T, Aihara A, et al. Clinical presentation of obturator hernia and review of the literature. Hernia. 2010;14:409-413.

[3] Petrie A, Tubbs RS, Matusz P, et al. Obturator Hernia: Anatomy, Embryology, Diagnosis, and Treatment. Clinical Anatomy. 2011;24:562569.

[4] Skandalakis LJ, Skandalakis PN, Gray SW, et al. Obturator hernia. Hernia.1995;4:425-439.

[5] Kulkarni SR, Punamiya AR, Naniwadekar RG, et al. Obturator hernia: A diagnostic challenge. Int JSurg Case Rep. 2013;4:606-608.

[6] Şenol K, Bayam ME, Duman U, et al. Challenging management of obturator hernia: a report of three cases and literature review. Ulus Travma Acil Cerrahi Derg. 2016;22:297-300.

[7] Mantoo SK, Mak K, Tan TJ. Obturator hernia: diagnosis and treatment in the modern era. Singapore Med J. 2009;50:866-870.

[8] Kammori M, Mafune K, Hirashima T, et al. Forty-three cases of obturator hernia. Am J Surg. 2004;187:549-552.

[9] Nasir BS, Zendejas B, Ali SM, et al. Obturator hernia: the Mayo Clinic experience. Hernia. 2012;16:315-319.

[10] Chang SS, Shan YS, Lin YJ, et al. A review of obturator hernia and a proposed algorithm for its diagnosis and treatment. World J Surg. 2005;29:450-454. 
[11] Köksal H, Ateş D, Nazik EE, et al. Predictive value of preoperative neutrophil-to-lymphocyte ratio while detecting bowel resection in hernia with iestinal incarceration. Ulus Travma Acil Cerrahi Derg. 2018;24:207210 .

[12] Xie X, Feng S, Tang Z, et al. Neutrophil-to-Lymphocyte Ratio Predicts the Severity of Incarcerated Groin Hernia. Med Sci Monit. 2017;23:55585563.

[13] Karadeniz E, Bayramoğlu A, Atamanalp SS. Sensitivity and Specificity of the Platelet-Lymphocyte Ratio and the Neutrophil-Lymphocyte Ratio in Diagnosing Acute Mesenteric Ischemia in Patients Operated on for the Diagnosis of Mesenteric Ischemia: A Retrospective Case-Control Study. J Invest Surg. 2019;19:1-8.

[14] Tanaka K, Hanyu N, Iida T, et al. Lactate levels in the detection of preoperative bowel strangulation. Am Surg. 2012;78:86-88.

[15] Luwaga RK, Galukande M, Owori FN. Serum lactate and phosphate as biomarkers of intestinal ischemia in a Ugandan tertiary hospital: a crosssectional study. Int J Emerg Med. 2013;6:44.

[16] Leow JJ, How KY, Goh MH, et al. Non-operative management of obturator hernia in an elderly female. Hernia. 2014;18:431-433.

How to cite this article: Semra Salimoğlu, Semra Demirli Atıc1. Prognostic Factors in Obturator Hernia. Signa Vitae. 2021;17(1):106111. doi:10.22514/sv.2020.16.0038. 